\title{
Changes to volumetric bone mineral density and bone strength after stroke: a prospective study
}

Karen Borschmann ${ }^{1,2 *}$, Marco YC Pang ${ }^{3}$, Sandra Iuliano ${ }^{4,5}$, Leonid Churilov ${ }^{2,4,6}$, Amy Brodtmann $^{2}$, Elif I. Ekinci ${ }^{4,5,7}$, Julie Bernhardt ${ }^{1,2}$

${ }^{1}$ School of Health Science, Latrobe University, Bundoora, Australia

${ }^{2}$ Stroke Division, Florey Institute of Neuroscience and Mental Health, University of Melbourne, Heidelberg, Australia

${ }^{3}$ Department of Rehabilitation Sciences, The Hong Kong Polytechnic University, Hung Hom, Hong Kong

${ }^{4}$ Department of Medicine, Austin Health, University of Melbourne, Heidelberg, Australia

${ }^{5}$ Endocrine Centre, Austin Health, Heidelberg, Australia

${ }^{6}$ Department of Mathematics and Statistics, University of Melbourne, Heidelberg, Australia

${ }^{7}$ Menzies School of Health Research, Darwin, Australia

*Corresponding author: karen.borschmann@florey.edu.au

Key words: bone, bone mineral density, bone structure, exercise, fracture, HR-pQCT, physical activity, stroke 


\begin{abstract}
Rationale and aim: Stroke survivors experience accelerated bone loss and increased fracture risk, particularly in paretic weight bearing limbs. Understanding how these changes unfold and their relationship to stroke severity and physical activity could help in the development of targeted interventions to prevent or reduce the severity of these outcomes. The primary aim of this study is to investigate the time course and magnitude of changes in volumetric bone mineral density (vBMD) within the first year after stroke, and to examine relationships with physical activity and motor recovery.
\end{abstract}

Design: Prospective, observational study of 43 non-diabetic, non-ambulant adults with first ever hemispheric stroke.

Primary outcome: difference in six month change of total vBMD between paretic and non-paretic distal tibiae, measured at $7 \%$ of bone length site using high-resolution peripheral quantitative computed tomography (HR-pQCT).

Secondary outcomes: Cortical and trabecular vBMD, cortical thickness, and total and cross-sectional areas of distal tibiae and radii of paretic and non-paretic limbs. Total body and regional BMD derived using dual-energy X-ray absorptiometry (DXA), physical activity measured using accelerometry, and motor recovery (Chedoke McMaster Stroke Assessment).

Discussion: Measuring the timing and magnitude of changes to vBMD and bone structure from immediately after stroke, and relationships between these changes with physical activity and motor recovery will provide the basis for targeted interventions to reduce fracture risk in stroke survivors. 


\section{Introduction}

Bone loss is accelerated during the first year after stroke. Reduced bone mineral density (BMD) is observed predominantly on the hemiparetic side (1). This increased bone loss partially accounts for an up to seven-fold increase in fracture risk compared with agematched controls $(2,3)$, with most post-stroke fractures occurring at the hip (4). Impaired mobility and prolonged immobilisation (5), motor impairment (6), muscle weakness (7), and reduced cardiovascular fitness (8) after stroke are associated with greater rates of bone loss. Physical activity in people with chronic stroke may enhance bone density and structure (9) but little is known about the relationship between physical activity and skeletal changes immediately after stroke.

Previous research of skeletal change after stroke has primarily utilised dual energy Xray absorptiometry (DXA) estimates of areal BMD (aBMD, expressed as $\mathrm{g} / \mathrm{cm}^{2}$ ) as an indirect measure of bone strength. Greater reductions in aBMD of paretic compared to non-paretic upper and lower limbs have been observed in longitudinal studies, particularly for people unable to walk after stroke $(5,10-12)$. Reduction of paretic limb aBMD within 12 months of stroke is up to 10 to $12 \%$ at the proximal femur and $17 \%$ at the humerus (11). Areal BMD is a two dimensional estimate of bone density. More accurate estimates of bone structure and distribution of its mineral content, which contribute to bone strength, are not possible using this technology. Peripheral computed quantitative tomography (pQCT) can provide estimates of volumetric bone mineral density (vBMD) and bone structure, which can be used to estimate bone strength. Compressive bone strength index (cBSI, total area $x$ total $\mathrm{vBMD}^{2,}, \mathrm{~g}^{2} / \mathrm{cm}^{4}$ ) is a noninvasive estimate of bone strength of the distal ends of long bones (13). $85 \%$ of the variance in failure load during mechanical compression tested on ex-vivo tibiae is predicted by cBSI (13). Volumetric BMD alone predicts $68 \%$ of this variance (13).

Low resolution pQCT was utilised in comparative studies of paretic and non-paretic upper and lower limbs after stroke (10, 14, 15). Pang et al. (2012) (15) in a cross sectional study of people with chronic stroke, observed reduced total vBMD at the site of $4 \%$ of radius length of paretic upper limbs (mean $321.7 \pm \mathrm{SD} 97.2 \mathrm{~g} / \mathrm{cm}^{3}$ ) compared 
to non-paretic upper limbs $\left(356.3 \pm 77.9 \mathrm{~g} / \mathrm{cm}^{3}, \mathrm{p}<0.03\right)$. In a longitudinal study, Lazoura et al. (10) observed significant reductions in trabecular and cortical vBMD in the radii of paretic and non-paretic limbs between 3 and 12 months after stroke. At the $4 \%$ radii site, the mean reduction in trabecular vBMD was $14.0 \%$ in paretic limbs and $6.8 \%$ in non-paretic limbs of males, and $9.3 \%$ and $7.3 \%$ respectively in females (SD not reported). At the $20 \%$ site, cortical vBMD reduction was $4.0 \%$ and $1.7 \%$ in males, and $2.6 \%$ and $1.2 \%$ in females. In the only pQCT study of tibiae after stroke known to the present authors, Pang et al.(2008) (14) undertook low resolution pQCT scans (in-plane pixel size $300 \mu \mathrm{m}$ ) at the site of $30 \%$ of tibia length in paretic and non-paretic legs of men $(n=30)$ and women $(n=25)$ who were more than a year post stroke. In the paretic legs of men and women they observed lower $(\mathrm{p}<0.05)$ cortical bone area (between limb difference for men $=1.5 \pm 6.0 \%$; women $4.5 \pm 6.8 \%)$, cortical bone mineral content $(1.9$ $\pm 5.8 \% ; 4.5 \pm 7.0 \%)$ and cortical thickness $(2.6 \pm 6.3 \% ; 3.6 \pm 8.4 \%)$, and reduced total bone area at this site in the paretic limbs of women $(2.8 \pm 4.2 \%)$. Little data exists describing the rates of change to bone structure and strength immediately and within the first year post stroke, and the relationship between these changes and physical activity levels during this period.

Other common sequelae to stroke and post-stroke immobility are loss of muscle mass $(16,17)$, hyperglycaemia and impaired insulin and glucose metabolism (18-20). Acute hyperglycaemia in non-diabetic people predicts increased risks of in-hospital mortality after ischemic stroke and poor functional recovery in stroke survivors $(19,20)$. Glucose metabolism after stroke is influenced not only by diabetes (diagnosed or undiagnosed) or an acute physiological 'stress response', but also site of brain lesion (21), muscle loss (16) and intramuscular changes (22). There is increased awareness of the role of the brain and neural processes in regulation of bone formation and resorption $(23,24)$ and glucose metabolism (25), however current knowledge of these relationships is not clear.

In this present prospective study we seek to further understand the acute and prolonged post-stroke changes that occur in vBMD and bone structure and their relationships with physical activity and motor recovery. We will utilise high-resolution pQCT, which enables high resolution imaging of bone (voxel size $82 \mu \mathrm{m}$ ), with minimal radiation 
exposure. HR-pQCT images allow for separate measurements of trabecular and cortical bone compartments and its resolution is high enough to assess the microarchitecture of trabecular bone. Volumetric BMD of paretic and non-paretic upper and lower limbs and physical activity will be assessed prospectively after stroke. It is hypothesised that within six months of stroke, vBMD will decline more in paretic limbs compared to nonparetic limbs. Other pre-specified data will be collected in order to explore relationships between post-stroke changes to bone, muscle, insulin and glucose metabolism, and physical activity to inform future studies.

\section{Methods}

\section{Design}

This is a single centre, prospective observational study. A pilot study $(n=10)$ was undertaken to determine feasibility and estimate the effect size of tibial vBMD (primary outcome). In the present study, participants will be assessed within two weeks of stroke, one month after T1, then at three, six and 12 months after stroke (Table 1).

The study is registered with the Australian New Zealand Clinical Trials Registry (ACTRN12612000123842). Ethics approval was granted by Austin Health and LaTrobe University Human Research Ethics Committees.

\section{Study Population}

Adults over the age of 40 years who are admitted to the Austin Hospital stroke unit (Heidelberg, Australia) within one week of hemispheric stroke onset, who are medically stable, unable to ambulate and able to follow simple verbal commands will be eligible. Exclusion criteria are known diabetes, history of stroke or other neurological disease, other conditions significantly limiting function (e.g. limb amputation) or use of medication known to alter bone metabolism (e.g. bisphosphonates).

\section{Pre-specified measures}

Demographics (age, gender, marital status, living arrangements, education level, smoking status), stroke characteristics (stroke severity via National Institute of Health 
Stroke Scale: NIHSS and stroke classification via Oxfordshire Classification), and past medical history including falls and previous bone fractures will be recorded.

\section{Outcomes}

\section{Primary outcome}

The primary outcome is difference in six month change of total vBMD between paretic and non-paretic distal tibiae. Total vBMD ( $\mathrm{mg}$ of hydroxyapatite $/ \mathrm{cm}^{3}$ ) will be measured at the left and right distal tibiae using HR-pQCT (Xtreme CT, Scanco Medical AG, Brüttisellen, Switzerland). Measurements will be at taken at $7 \%$ of bone length measured from the distal end; a region containing both trabecular and cortical bone. Reliability of this measure, coefficient of variation (CV), is $1.3 \%$ (26). Scans cannot be undertaken if metal is within the scanning region. Images will be excluded if movement occurs during scanning.

\section{Secondary outcomes}

Trabecular and cortical bone density and structure: vBMD (cortical and trabecular), cortical thickness, and total and cortical cross sectional areas will be measured at the left and right distal tibiae (7\% site) and distal radii (4\% site) using HR-pQCT. Trabecular number $(\mathrm{TbN})$ as the inverse of the mean distance between mid-axes of trabeculae, will be derived by HR-pQCT software; trabecular thickness (TbTh, $\mu \mathrm{m})$ and separation (TbSp, $\mu \mathrm{m})$ will be derived from $\mathrm{TbN}$ and trabecular vBMD $(27,28)$, and distribution of separation ( TbSp SD $\mu \mathrm{m}$ ) will be calculated. CV for these parameters ranges from $0.7 \%$ to $4.4 \%$ (26).

Areal bone mineral density and body composition: Total body and regional BMD, and lean and fat mass will be determined using DXA (DPX-L, version 1.3z: Lunar Madison, WI), CV $=1-1.2 \%$ (29). All scans will be performed by the same technician using the same scanner for consecutive assessments.

Physical activity will be measured using a single-axis accelerometer with switch tilt, sample rate $10 \mathrm{~Hz}$ (PAL2, positional activity logger 2, Gorman ProMed Pty Ltd, 
Melbourne, Australia) (30). PAL2 registers the number of changes in position and the amount of time that participants spend lying down, sitting, standing and walking. There is high agreement between PAL2 recordings and labour intensive "gold standard" behavioural mapping (31). Physical activity is consistent across days in acute stroke patients (32). The device will be worn for one day from $8 \mathrm{am}$ to $5 \mathrm{pm}$ at each time point, which represents the most active part of the day for people with acute stroke (33).

Motor impairment: the Chedoke McMaster Stroke Assessment will be used to evaluate the severity of motor impairment in stroke affected upper and lower limbs (34).

\section{Exploratory measures}

To allow for future exploration of links between bone formation and glucose metabolism the following additional measures will be included:

1. Glucose metabolism: fasting glucose and insulin concentrations will be measured. Insulin sensitivity will be calculated using the homeostasis model assessment (HOMA) index (35). Two hour 75 gram oral glucose tolerance test (OGTT) will be performed to observe changes in glucose metabolism over time. Glycated haemoglobin $\left(\mathrm{HbA}_{1 \mathrm{c}}\right)$ will be measured to identify the average plasma glucose concentration during the preceding three months. Plasma glucose level will be measured using a hexokinase assay on the Roche Cobas 702. Insulin will be measured by electrochemiluniscence immunoassay (ECLIA) on the Roche Cobas 602. HbA1c will be measured by immunoassay on the Roche Integra 800 .

2. Bone formation and resorption markers will be measured to observe changes in bone turnover. Formation markers: Osteocalcin, N-terminal propeptide of type 1 procollagen (PINP); and resorption marker: serum $\mathrm{C}$ terminal telopeptide of types 1 collagen (CTX) will be determined by electrochemiluminenescence immunoassay (Elecsys 1010 Analytics, Roche Diagnostics, Germany, intra- and inter-assay CV 3-8\%) (36).

Fasting blood samples will be taken before 9am, and will be batch analysed. 


\section{Sample size}

The pilot phase of this study $(n=10)$ was used to estimate effect size, using STATA statistical software. Assuming one sample, 2-tailed test with criterion for significance of $\mathrm{p}=0.05$, and using the difference in six-month change between paretic and non-paretic limbs in distal tibia vBMD [mean reduction paretic limb $=-1.43 \%$, SD 1.92, non-paretic $\operatorname{limb}=-0.45 \%$, SD 1.37; effect size 0.59], a sample size of 25 participants will provide $80 \%$ power to detect a significant effect if present. Adjusting for expected mortality of $30 \%$ within the first year of stroke (37), 33 participants are required. Final results will pool data from the pilot phase $(n=10)$ with results of the present study $(n=33)$, giving a total reported sample of 43 participants.

\section{Statistical analysis}

We will observe the interaction between time and bone changes in the two lower limbs (i.e., do paretic and non-paretic limbs experience the same decline in vBMD over time?). Comparison of change in paretic and non-paretic limbs will be assessed using a one sample t-test, subject to the validity distribution assumptions, to compare difference in change over time between paretic and non-paretic limb total tibia vBMD. Multilevel analysis with patient as a level will be undertaken to model change over time in primary and secondary outcomes using random effects generalised least squares regression and/ or generalised estimating equations. Additionally, the relationships between primary outcomes and covariates (i.e. physical activity, motor recovery, muscle mass) will be assessed using linear regression, adjusting for gender, age and initial stroke severity. Participants act as their own control between paretic and non-paretic limbs, thus those with missing data will be excluded from analysis. This will not affect internal validity, but will affect generalisability of results. Statistical analysis will be performed using Stata statistical software. A significance level of 0.05 will be set for all statistical tests.

\section{Summary}

Bone fracture is a common and serious sequelae following stroke. High resolution pQCT will be utilised for the first time in this study to prospectively examine changes in vBMD and bone structure of tibiae and radii from within two weeks of stroke. 
Relationships to physical activity and motor recovery will be investigated. Total body and regional muscle mass derived from DXA, indices of insulin and glucose metabolism and blood markers of bone turnover will be assessed concomitantly in order to inform future studies. Knowledge of interactions between these factors from early after stroke will provide scientific evidence to develop specific and targeted rehabilitation programs, in order to improve quality of life and health outcomes of stroke survivors.

\section{Acknowledgments}

We acknowledge the assistance of Dr Ali Ghasem-Zadeh. We thank the Victorian State Government for operational infrastructure support. This study is funded by the Australian Research Council (ARC-FT09901086), Austin Health Medical Research Fund and LaTrobe University Faculty Research Grant. EIE is supported by an NHMRC Early Career Fellowship (APP\#APP1054312) and AB by an NHMRC Career Development Award. 


\section{References}

1. Jorgensen L, Jacobsen BK. Changes in muscle mass, fat mass, and bone mineral content in the legs after stroke: A 1 year prospective study. Bone. 2001;28(6):655-9.

2. Kanis J, Oden A, Johnell O. Acute and long-term increase in fracture risk after hospitalization for stroke. Stroke. 2001;32(3):702-6.

3. Dennis MS, Lo KM, McDowall M, West T. Fractures after stroke: frequency, types, and associations. Stroke. 2002;33(3):728-34.

4. Ramnemark A, Nyberg R, Borssen B, Olsson T, Gustafson Y. Fractures after stroke. Osteoporos Int. 1998;8:92-5.

5. Jorgensen L, Jacobsen BK, Wilsgaard T, Magnus JH. Walking after stroke: Does it matter? Changes in bone mineral density within the first 12 months after stroke. A longitudinal study. Osteoporosis International. 2000;11(5):381-7.

6. Hachinski V, Donnan GA, Gorelick PB, Hacke W, Cramer SC, Kaste M, et al. Stroke: working toward a prioritized world agenda. Int J Stroke. 2010;5(4):238-56. Epub 2010/07/20.

7. Ashe M, Fehling, Eng J, Khan K, McKay H. Bone geometric response to chronic disuse following stroke: A pQCT study. Journal of Musculoskeletal Neuronal Interactions. 2006;6(3):226-33.

8. Pang M, Eng J, McKay H, Dawson A. Reduced hip bone mineral density is related to physical fitness and leg lean mass in ambulatory individuals with chronic stroke. Osteoporosis International. 2005;16(12):1769-79.

9. Pang M, Ashe M, Eng J, McKay H, Dawson A. A 19-week exercise program for people with chronic stroke enhances bone geometry at the tibia: a peripheral quantitative computed tomography study. Osteoporosis International. 2006;17(11):1615-25.

10. Lazoura O, Groumas N, Antoniadou E, Papadaki PJ, Papadimitriou A, Thriskos P, et al. Bone Mineral Density Alterations in Upper and Lower Extremities 12 Months After Stroke Measured by Peripheral Quantitative Computed Tomography and DXA. Journal of Clinical Densitometry. 2008;11(4):511-7.

11. Ramnemark A, Nyberg L, Lorentzon R, England U, Gustafson Y. Progressive hemiosteoporosis on the paretic side and increased bone mineral density in the nonparetic arm the first year after severe stroke. Osteoporosis International. 1999;9(3):269-75.

12. Liu M, Tsuji T, Higuchi Y, Domen K, Tsujiuchi K, Chino N. Osteoporosis in hemiplegic stroke patients as studied with dual-energy X-ray absorptiometry. Archives of Physical Medicine and Rehabilitation. 1999;80(10):1219-26.

13. Kontulainen SA, Johnston JD, Liu D, Leung C, Oxland TR, McKay HA. Strength indices from pQCT imaging predict up to $85 \%$ of variance in bone failure properties at tibial epiphysis and diaphysis. J Musculoskelet Neuronal Interact. 2008;8(4):401-9. Epub 2009/01/17.

14. Pang MYC, Ashe MC, Eng JJ. Tibial Bone Geometry in Chronic Stroke Patients: Influence of Sex, Cardiovascular Health, and Muscle Mass. Journal of Bone \& Mineral Research. 2008;23(7):1023-30.

15. Pang MY, Cheng AQ, Warburton DE, Jones AY. Relative impact of neuromuscular and cardiovascular factors on bone strength index of the hemiparetic distal radius epiphysis among individuals with chronic stroke. Osteoporos Int. 2012;23(9):2369-79. Epub 2012/02/09. 
16. English C, McLennan H, Thoirs K, Coates A, Bernhardt J. Loss of skeletal muscle mass after stroke: a systematic review. International Journal of Stroke. 2010;5(5):395-402.

17. Booth F, Gollnick P. Effects of disuse on the structure and function of skeletal muscle. Med Sci Sports Exerc. 1983;15(5):415-20.

18. Hafer-Macko C, Ryan A, Ivey F, Macko R. Skeletal muscle changes after hemiparetic stroke and potential beneficial effects of evercise intervention strategies. Journal of Rehabilitation Research and Development. 2008;45(2):261-72.

19. Weir CJ, Murray GD, Dyker AG, Lees KR. Is hyperglycaemia an independent predictor of poor outcome after acute stroke? Results of a long term follow up study. Brit Med J. 1997;314(7090):1303-6.

20. Capes SE, Hunt D, Malmberg K, Pathak P, Gerstein HC. Stress Hyperglycemia and Prognosis of Stroke in Nondiabetic and Diabetic Patients: A Systematic Overview. Stroke. 2001;32(10):2426-32.

21. Allport L, Butcher K, Baird T, Colman P, MacGregor L, Tress B, et al. Acute stress hyperglycemia is associated with insular cortical ischemia. Stroke. 2004;35(1):248-.

22. Ivey F, Ryan A, Hafer-Macko C, Garrity B, Sorkin J, Goldberg A, et al. High prevalence of abnormal glucose metabolism and poor sensitivity of fasting plasma glucose in the chronic phase of stroke. Cerebrovascular Diseases. 2006;22:368-71.

23. Driessler F, Baldock PA. Hypothalamic regulation of bone. J Mol Endocrinol. 2010;45(4):175-81. Epub 2010/07/28.

24. Jiang S, D, Shen C, Jiang L, S, Dai L, et al. Differences of bone mass and bone structure in osteopenic rat models caused by spinal cord injury and ovariectomy. Osteoporosis International. 2007;18(6):743-50.

25. Ferron M, Wei J, Yoshizawa T, Del Fattore A, DePinho RA, Teti A, et al. Insulin signaling in osteoblasts integrates bone remodeling and energy metabolism. Cell. 2010;142(2):296-308. Epub 2010/07/27.

26. Boutroy S, Bouxsein ML, Munoz F, Delmas PD. In Vivo Assessment of Trabecular Bone Microarchitecture by High-Resolution Peripheral Quantitative Computed Tomography. Journal of Clinical Endocrinology \& Metabolism. 2005;90(12):6508-15.

27. Laib A, Hauselmann HJ, Ruegsegger P. In vivo high resolution 3D-QCT of the human forearm. Technol Health Care. 1998;6(5-6):329-37. Epub 1999/04/01.

28. Laib A, Ruegsegger P. Comparison of structure extraction methods for in vivo trabecular bone measurements. Comput Med Imaging Graph. 1999;23(2):69-74. Epub 1999/05/05.

29. Baim S, Wilson C, Lewiecki E, Luckey M, Downs R, Lentle B. Precision Assessment and Radiation Safety for Dualenergy Xray Absorptiometry (DXA). Journal of Clinical Densitometry (JCD). 2005;8(4):371-8.

30. Diggory P, Gorman M, Schwarz J, Helme R. An automatic device to measure time spent upright. Clinical Rehabilitation. 1994;8(4):353-7.

31. Kramer S, Cumming T, Churilov L, Bernhardt J. Measuring activity levels at an acute stroke ward: comparing observations to a device. Biomed Research International. 2013;2013(Article ID 460482):8 pages.

32. Rantalainen T, Nikander R, Daly RM, Heinonen A, Sievanen H. Exercise loading and cortical bone distribution at the tibial shaft. Bone.2011 Apr 1;48(4):786-91. doi(2010 Nov 30):10.1016/j.bone.2010.11.013. 
33. Bernhardt J, Dewey H, Thrift A, Donnan G. Inactive and alone. Physical activitiy within the first 14 days of acute stroke unit care. Stroke. 2004;35:1005-9.

34. Gowland C, Stratford P, Ward M, Moreland J, Torresin W, Van Hullenaar S, et al. Measuring physical impairment and disability with the Chedoke-McMaster Stroke Assessment. Stroke. 1993;24(1):58-63.

35. Matthews D, Hosker J, Rudenski A, Naylor B, Treacher D, Turner R. Homeostasis model assessment: insulin resistance and B-cell function from fasting plasma glucose and insulin concentrations in man. Diabeologia. 1985;28:412-9.

36. Bjornerem A, Ghasem-Zadeh A, Bui M, Wang X, Rantzau C, Nguyen TV, et al. Remodeling markers are associated with larger intracortical surface area but smaller trabecular surface area: a twin study. Bone. 2011;49(6):1125-30. Epub 2011/08/30.

37. Thrift AG, Dewey HM, Macdonell RA, McNeil JJ, Donnan GA. Stroke incidence on the east coast of Australia: the North East Melbourne Stroke Incidence Study (NEMESIS). Stroke. 2000;31(9):2087-92. Epub 2000/09/08. 
Table 1: Time points of participant assessment

\begin{tabular}{|c|c|c|c|c|c|}
\hline Outcome Measure & Baseline* & $\begin{array}{c}1 \\
\text { month }\end{array}$ & $\begin{array}{c}3 \\
\text { month }\end{array}$ & $\begin{array}{c}6 \\
\text { month }\end{array}$ & $\begin{array}{c}12 \\
\text { month }\end{array}$ \\
\hline HR-pQCT & $\checkmark$ & $\mathrm{x}$ & $\mathrm{x}$ & $\checkmark$ & $\checkmark$ \\
\hline DXA & $\checkmark$ & $\checkmark$ & $\checkmark$ & $\checkmark$ & $\checkmark$ \\
\hline Activity monitoring & $\checkmark$ & $\checkmark$ & $\checkmark$ & $\checkmark$ & $\checkmark$ \\
\hline OGTT & $\checkmark$ & $\mathrm{x}$ & $\mathrm{x}$ & $\checkmark$ & $\checkmark$ \\
\hline $\begin{array}{l}\text { Fasting glucose, insulin \& bone } \\
\text { turnover markers }\end{array}$ & $\checkmark$ & $\checkmark$ & $\checkmark$ & $\checkmark$ & $\checkmark$ \\
\hline $\mathrm{HbA1c}$ & $\checkmark$ & $\mathrm{x}$ & $\checkmark$ & $\checkmark$ & $\checkmark$ \\
\hline Motor impairment & $\checkmark$ & $\checkmark$ & $\checkmark$ & $\checkmark$ & $\checkmark$ \\
\hline
\end{tabular}

*Within two weeks of stroke; DXA = dual energy X-ray absorptiometry; HbA1c= glycated haemoglobin; HR-pQCT = high resolution peripheral computed tomography; OGTT $=$ two hour oral glucose tolerance test 